\title{
Verlagswechsel und Reduzierung des Abonnementspreises ab 2004
}

\author{
Liebe Leserinnen, liebe Leser,
}

Ab dem Jahre 2004 wird die «Analytische Psychologie» in einem neuen Verlag herauskommen: im Verlag Brandes \& Apsel in Frankfurt a.M.

Vorausgegangen waren dieser Entscheidung lange und intensive Diskussionen der Herausgeber und der Redaktion sowohl untereinander als auch mit Herrn Dr. h.c. Thomas Karger. Nachdem seit etlichen Jahren die Zahl der Abonnenten kontinuierlich zurückgegangen war, mussten wir uns überlegen, wie wir dem begegnen können. Neben der allgemeinen schwierigen wirtschaftlichen Situation, die die meisten Fachzeitschriften trifft, scheinen aus unserer Sicht vor allem drei Faktoren für den Rückgang der Abonnentenzahl verantwortlich zu sein: Der vergleichsweise sehr hohe Preis der Zeitschrift, inhaltliche Schwächen und das marketingtechnische Problem, dass wir im Karger Verlag, mit seinen hochspezialisierten Publikationen für ein medizinisch-naturwissenschaftliches, vor allem forscherisch und akademisch arbeitendes Lesepublikum, nicht im idealen publizistischen Kontext präsent sind.

Obwohl der Karger Verlag bereit war, uns finanziell grosszügig entgegenzukommen, haben wir uns entschlossen, zu einem Verlag zu wechseln, der im Bereich der Psychotherapie und Psychoanalyse publizistisch präsent ist.

Es ist uns nach fast 30 Jahren sehr guter Zusammenarbeit nicht leicht gefallen, uns vom Karger Verlag zu trennen, der unsere Zeitschrift stets nachhaltig unterstützt hat und einen persönlich wie fachlich aussergewöhnlich guten Umgang mit uns pflegte. Eine Würdigung der besonderen Rolle von Herrn Dr. h.c. Karger für die Zeitschrift wird in einem der nächsten Hefte erscheinen. An dieser Stelle möchten wir uns vor allem bei den Verlagsmitarbeiterinnen und -mitarbeitern sehr herzlich bedanken.

Wir hoffen, dass Sie uns auch weiterhin die Treue halten, wenn wir ab $2004 \mathrm{im}$ Verlag Brandes \& Apsel erscheinen werden. Der Preis des Abonnements wird bei $€$ 59.inklusive Versand liegen, was einer Halbierung des bisherigen Abonnementspreises entspricht; die Zeitschrift wird auch etwas umfangreicher werden.

Redaktionell sind wir in einem Umstrukturierungsprozess begriffen, der auch die Ziele und Inhalte der Zeitschrift betrifft. Wir haben uns vorgenommen, wieder mehr Abonnenten aus den jungianischen Fachgesellschaften, aber auch jene Psychotherapeuten und Psychoanalytiker für die Zeitschrift zu gewinnen, die an den unterschiedlichen Strömungen innerhalb der analytischen Psychologie und an einem Dialog mit den anderen Traditionen der psychotherapeutischen und psychoanalytischen Gemeinschaft interessiert sind.

Gustav Bovensiepen

\section{KARGER}

Fax +4161306 1234

E-Mail karger@karger.ch

www.karger.com

() 2003 S. Karger AG, Basel

0301-3006/03/0343-0155\$19.50/0 